\title{
Antibiotic resistance and the evolution of bacteria
}

\section{from Mark Richmond}

A PAPER by Victoria Hughes and Naomi Datta in this week's issue of Nature (p.725) fills a gap in our knowledge of the emergence of antibiotic resistance in bacteria. The study makes use of a remarkable collection of bacteria made between 1917 and 1954 - before the use of antibiotics became widespread and at a time when little was known of bacterial genetics - and kept in sealed vessels since that period.

The study of bacterial plasmids and episomes, vital to an understanding of the spread of antibiotic resistance, did not really begin until the 1960 s. Early on, most experimental work was focused on a few bacterial species - notably Escherichia coli and Staphylococcus aureus - and the plasmids/episomes involved were limited to a few types of phage and antibioticresistance factors and to some colicin factors. What emerged was a picture of the bacterial genome in which most of the genetic information in the organism was seen to be concentrated as a single large circular DNA molecule - the chromosome - of molecular weight about $2 \times 10^{9}$, but some was carried as small circular DNA molecules which did not seem absolutely necessary for bacterial growth and survival in most environmental circumstances. When these extrachromosomal DNA elements carried the relevant genetic information they behaved as bacterial viruses, capable of being triggered to multiply rapidly to burst the host cell.

A further characteristic of many plasmids and phage was their ability to transfer from one bacterial strain or species to another and, in some cases, to catalyse the transfer of further independent circular DNA molecules. At the extreme, certain bacterial plasmids, following integration into the bacterial chromosome, could even cause the transfer of a copy of a whole chromosome from one bacterial cell to another to give rise, at least transiently, to a diploid organism.

Our awareness of the range of possible interactions of bacterial plasmids and the effects these interactions had on the bacteria radically altered our view of the individuality of single bacterial cells. Populations of bacteria could now be seen as a single gene pool, with most of the information segregated for much of the time in the individual members of the population, but with extra DNA molecules distributed

Mark Richmond is Vice-Chancellor and Professor of Molecular Microbiology in the University of Manchester, Manchester M13 9PL. within the population and capable of being transferred within the members of the population. Furthermore, that integration and excision of plasmids into and from the chromosome sometimes transferred blocks of information from the chromosomal to the extrachromosomal state underlined the fact that a bacterial population as a whole had considerably genetic fluidity even if, under normal circumstances, this fluidity was constrained. When it was realized that this potential fluidity extended to populations containing different species and genera it was clear that one of the main processes determining the means of bacterial evolution had been discovered and that a much deeper understanding of the emergence of antibiotic resistance could be gained.

The emergence of resistance had already been broadly defined: the introduction of a novel antibacterial agent was followed by the rapid appearance of resistant bacterial populations which then became more and more widespread, both geographically and also with respect to the bacterial strains and species exhibiting resistance, as the use of the agent continued.

It became clear that two processes are involved in the evolution of resistant populations: the 'invention' of the resistance genes themselves, and their multiplication and spread. Relatively little is known even now about the first of the two stages, though there are some clues. As for the second, the mechanisms of genetic exchange discussed above, coupled with selection, can provide a complete explanation.

One part of this story has, however, always been taken for granted, and it is here that the paper appearing in this week's issue of Nature finally provides definitive evidence. For the scenario outlined above to be correct, bacterial strains isolated before the advent of large-scale use of antibiotics should exhibit all the genetic flexibility of the antibiotic-resistant bacteria we see nowadays, but the incidence of resistance genes themselves should be low. Using a large collection of Gram-negative bacterial isolates made by R.G.E. Murray from before the antibiotic era, Naomi Datta and Victoria Hughes have now shown that this is indeed the case.

In a sense, therefore, Murray's collection is representative of the kinds of bacterial population that existed when antibiotic therapy started in the early 1940s. Plasmid transfer and gene exchange supported by appropriate pressure have done the rest.

\section{Human genetics}

\section{Is there a human $T / t$ locus?}

from P.N. Goodfellow and P.W. Andrews

CHROMOSOME 17 of wild mice frequently contains a region of mutant genes, known as the $T / t$ complex, maintained in association by local suppression of recombination. Within the region, which stretches from the centromere to beyond the $\mathrm{H}-2$ locus, different $t$-haplotypes are characterized by different $\boldsymbol{H}-2$ types and different recessive lethal mutations with striking effects on embryogenesis ${ }^{1}$. The $t$-haplotypes also carry a group of interesting mutations which, when homozygous, cause male sterility and when heterozygous cause carrier males to transmit the chromosome carrying the $t$-haplotype to nearly 100 per cent of their offspring. A similar constellation of mutations known as Segregation Distorter has been observed in wild populations of Drosophila melanogaster. Evidence for a locus homologous to the $T / t$ locus in mammals other than mice, and perhaps rats ${ }^{2}$, has been harder to find. Several recent papers suggest that a human homologue of the $T / t$ complex may indeed exist ${ }^{3-6}$.

A human equivalent of the murine $T / t$ complex would be expected to be associated with segregation distortion, recombination suppression and recessive lethality. It might also be expected that an equivalent of the $T / t$ complex would be linked to the human equivalent of $\mathrm{H}-2$, $H L A$. This possibility has provided the starting point for several searches for the human $T / t$ complex. Genes known to be linked to the $H L A$ region include loci controlling class 1 and class 2 cell-surface antigens, loci controlling complement components and a locus controlling the enzyme glyoxalase $(G L O)$. At least two alleles are known for each of the four major histocompatibility complex (MHC)-linked complement loci, but these are so closely linked that recombination between them has not been observed and only 14 common cis combinations of alleles (complotypes) occur.

In a survey of families drawn from the Boston area, Awdeh eh al. ${ }^{3}$ observed that several complotypes showed non-random association with alleles at the $H L A-D$ and $H L A-B$ loci and less often with alleles of

P. N. Goodfellow is in the Laboratory of Human Molecular Genetics, Imperial Cancer Research Fund, Lincoln's Inn Fields, London WC2A $3 P X$ and P.W. Andrews in the Wistar Institute, 36th Street and Spruce, Philadelphia, Pennsylvania 19104. 